\title{
The Constitution of Group Cognition
}

\author{
Gerry Stahl \\ Drexel University, Philadelphia, USA \\ Gerry@GerryStahl.net
}

\begin{abstract}
Cognition is no longer confined to the solitary musings of an armchair philosopher, but takes place, for instance, in problem-solving efforts of teams of people distributed around the world and involving various artifacts. The study of such cognition can unfold at multiple units of analysis. Here, three cases of problem solving by virtual math teams demonstrate the mix of individual, group and social levels of cognition. They show how a resource like a mathematical topic can bridge the different levels. Focusing on the under-researched phenomena of group cognition, the presentation highlights three pre-conditions for the constitution of group cognition: longer sequences of responses, persistent co-attention and shared understanding. Together, these structure a virtual analog of physical embodiment: being-there-together, where what is there is understood as co-experienced.
\end{abstract}

\section{COGNITION AT MULTIPLE LEVELS}

There is a venerable tradition in philosophy that cognition is a mysterious faculty of individual human beings. Increasingly since the late nineteenth century, it has become clear that even when thoughts appear to be expressed by an individual they are the product of more complex factors. Cognitive abilities and perspectives develop over time through one's embeddedness in a physical, social, cultural and historical world. Thinking is closely related to speaking, a form of communication with others. Particularly in our technological world, thinking is mediated by a broad variety of artifacts and by other features of the context in which we are situated.

Rather than thinking about thinking, I try to explore cognition by generating data in which one can observe cognitive processes at work (Stahl, 2006; 2009; 2013). I do this by having small groups of students collaborate on mathematical problems in a setting where their whole interaction can be captured. The motivation for this approach is the theory of Vygotsky, the sociocultural psychologist who proposed that higher-level human mental abilities are acquired first in small-group interactions. In exploring such group cognition, I have found that there is a rich interplay of processes at individual, small-group and community levels of cognitive processing.

In the following, I will summarize three case studies in order to illustrate how cognitive processes at multiple levels can work together. In the first case, two students solve a high-school math problem that has stumped them for some time. The problem-solving steps the dyad go through as a team are typical for how proficient students solve problems individually. In the discourse captured in this case, one can see how the group integrates contributions from the two 
individual participants to accomplish a task in accordance with community standards of practice - illustrating the productive interplay of cognitive levels. The sequence of ten discourse moves by the group details their extended sequential approach to the problem. In the second study, three students develop techniques for helping each other to see what they are seeing in the diagram they have drawn for a math problem. This persistent co-attention to a shared object of analysis allows the team to solve their problem as a group. Similarly in the third example, the students are able to work together because they effectively manage their shared understanding of the problem.

I propose that it is often fruitful to analyze cognition on multiple levels and that the processes at the different levels work together. A variety of interactional resources are typically at work bridging the levels. In the three illustrative case studies, topics in high-school mathematics centrally figure as resources that bring together individual, small-group and community cognitive processes.

\section{VIRTUAL MATH TEAMS}

The study of group cognition requires careful review and analysis of all the interaction within a group during the achievement of a cognitively significant task, such as solving a challenging problem. I have arranged for this by designing an online software environment in which several people can meet and interact effectively to solve math problems. This Virtual Math Teams (VMT) environment supports synchronous text chat and a shared whiteboard for drawing figures (Stahl, 2009). Recently, it has been expanded to incorporate a multi-user version of dynamic geometry, in which geometric figures can be interactively constructed and dynamically dragged (Stahl, 2013). The software is instrumented to capture all interaction and to allow it to be displayed, replayed and analyzed. This avoids the many problems of audio and video recording in classrooms. Students communicate online, avoiding the interpretational issues of eye gaze, bodily gesture and vocal intonation. When possible, groups are composed of students who do not know each other outside of the online setting, so that researchers reviewing a record of interaction can know everything about the participants and their background knowledge that the participants know about each other. Since group cognition is defined as consisting of those knowledge-building or problem-solving processes that take place in the group interaction (Stahl, 2006), the VMT environment can capture a complete history of group-cognitive events.

When a group enters the VMT environment, it is presented with a challenging math problem, designed to guide the group interaction in an academically productive direction. The problem acts as a resource for the group. The group must interpret the problem statement, elaborate the way in which it wants to conceive the problem and determine how to proceed. A math problem can serve as an effective interactional resource for bridging across cognitive levels. Typically, it introduces content - definitions, elements, procedures, principles, practices, proposals, theorems, questions - from the cultural traditions of mathematics and from school curriculum. In so doing, it recalls or stimulates individual cognitive responses-memories, skills, knowledge, calculations, deductions. It is then up to the group interaction to bring these together, to organize the individual contributions as they unfold in the ongoing interaction in order to achieve the goals called for by the community, institutional, disciplinary and historical sources. In this way, the group interaction may play a central role in the multilevel cognition, interpreting, enacting 
and integrating elements from the other levels, producing a unified cognitive result and thereby providing a model for future community practice or individual skill.

It may seem ironic that an online environment has been selected for the empirical study of how cognition is "embodied" in group interactions and community contexts. In the VMT environment, participants are not physically present to each other. They do not see interactional contributions being produced by individuals. Rather, text chat postings suddenly appear as complete units on the screen and geometric elements are drawn or dragged without visible hands manipulating them. As we will see below, Aznx does not see how Bwang is gradually putting together and occasionally repairing a sentence to be posted. Jason cannot follow Qwertyuiop's gaze to see where his attention is focused. Yet, there are some elements of embodiment, at least virtually. Each participant is represented in the VMT interface with a login handle, associated with their chat postings. There are awareness notices indicating who is typing a pending chat contribution or who is engaged in a geometric construction action. The software interface presents a complexly structured visual manifold. Students quickly develop online practices to adapt to the new environment, to overcome the limitations of the media and to implement alternative means for missing abilities, as seen in the following case studies. Within this computer-mediated context, individual and group levels of cognition are focused on situated entities from specific perspectives; multilevel cognition is embodied in an intersubjective world.

\section{CONSTRUCTING DIAMONDS}

Cognition is neither a unitary phenomenon nor a temporally fixed one. Hegel described the logical stages involved in the development of cognition in his Phenomenology of Mind (1807/1967). Vygotsky explored the development of a person's cognition through psychological experiments reported in Mind in Society (1930/1978), emphasizing the priority of intersubjective group cognition:

Every function in the child's cultural development appears twice: first, on the social level, and later, on the individual level; first, between people (interpsychological), and then inside the child (intrapsychological). This applies equally to voluntary attention, to logical memory, and to the formation of concepts. All the higher [human mental] functions originate as actual relations between human individuals. (p. 57, emphasis in original)

Research on computer-supported collaborative learning (CSCL) (Stahl, Koschmann \& Suthers, 2013) can make visible the development and the unfolding of cognitive functions in small groups, shedding light on the less-visible processes that can subsequently be carried out by people individually or "internally." A research method for undertaking such analysis is suggested by the field of conversation analysis (CA) (Sacks, 1962/1995). CA was inspired by ethnomethodology, a sociological approach focused on describing the "work" that people typically do in interactions with others to establish social order and to construct meaning (Garfinkel, 1967). CA applies this approach to analyzing everyday conversation. A central finding of $\mathrm{CA}$ is that the work of conversation is accomplished through the sequential construction of "adjacency pairs," short sequences in which one person's utterance elicits a response in the form of a following utterance by an interlocutor-for instance a question-answer pair. In looking for examples of mathematical problem solving by groups, we are more interested 
in "longer sequences," in which a series of adjacency pairs are constructed to accomplish the larger cognitive goal.

Longer sequences have only been suggested in CA (Sacks, 1962/1995, Vol. 2, p. 354; Schegloff, 2007, pp. 12, 213), not extensively analyzed. In the final excerpt from a VMT interaction among three students, I analyzed their successful problem-solving effort as a longer sequence, consisting of ten discourse moves, each linguistically organized as an adjacency pair (Stahl, 2011). I treated their four-hour-long online interaction in terms of a temporal hierarchy of: a group event, four scheduled sessions, several conversational topics, many discourse moves, adjacency pairs, textual utterances and indexical references. In the first session, the students had been asked to work on a topic in mathematical combinatorics, determining the number of squares and composite sticks needed to build a stair-step pattern at different stages of growth. By the fourth session, the students had set themselves the topic of analyzing a diamond pattern, illustrated by them at stages $n=2$ and $n=3$ in the screen image of the VMT software interface in Figure 1.

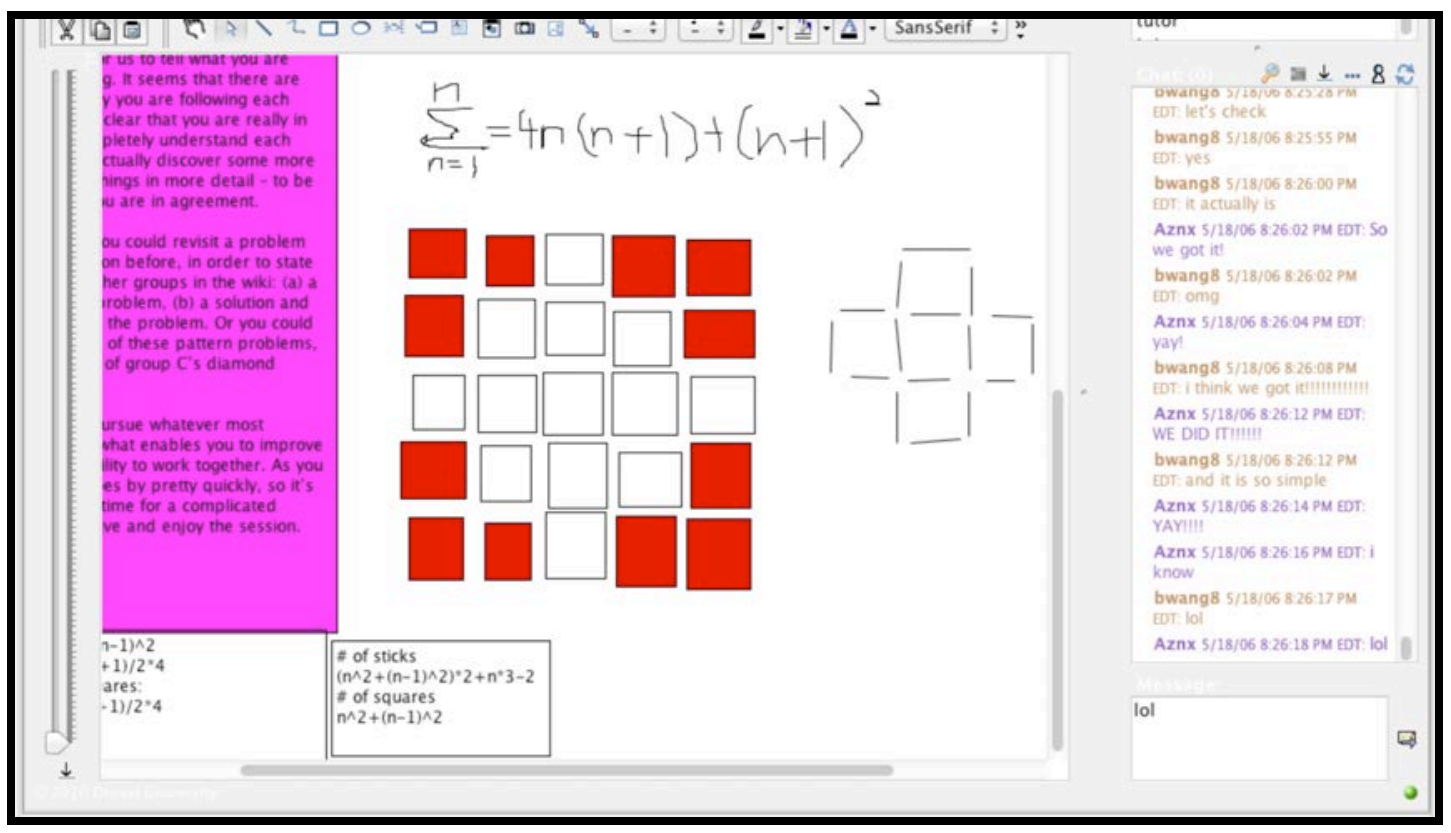

Figure 1. Discussion and drawings of diamond pattern.

In their final conversational topic, two students with login names of Bwang and Aznx decide to try again to solve this problem, despite not being able to do so for the past two hours and despite the fact that their scheduled online time is already over. In the course of ten minutes, 100 chat lines of text are posted. The analysis highlights ten adjacency pairs that were central to this discourse. Each adjacency pair is listed in Log 1, under an added descriptive heading. Although there is not space here to provide the full chat or a complete analysis, this selection from the interaction should give a sense of the problem-solving process. 


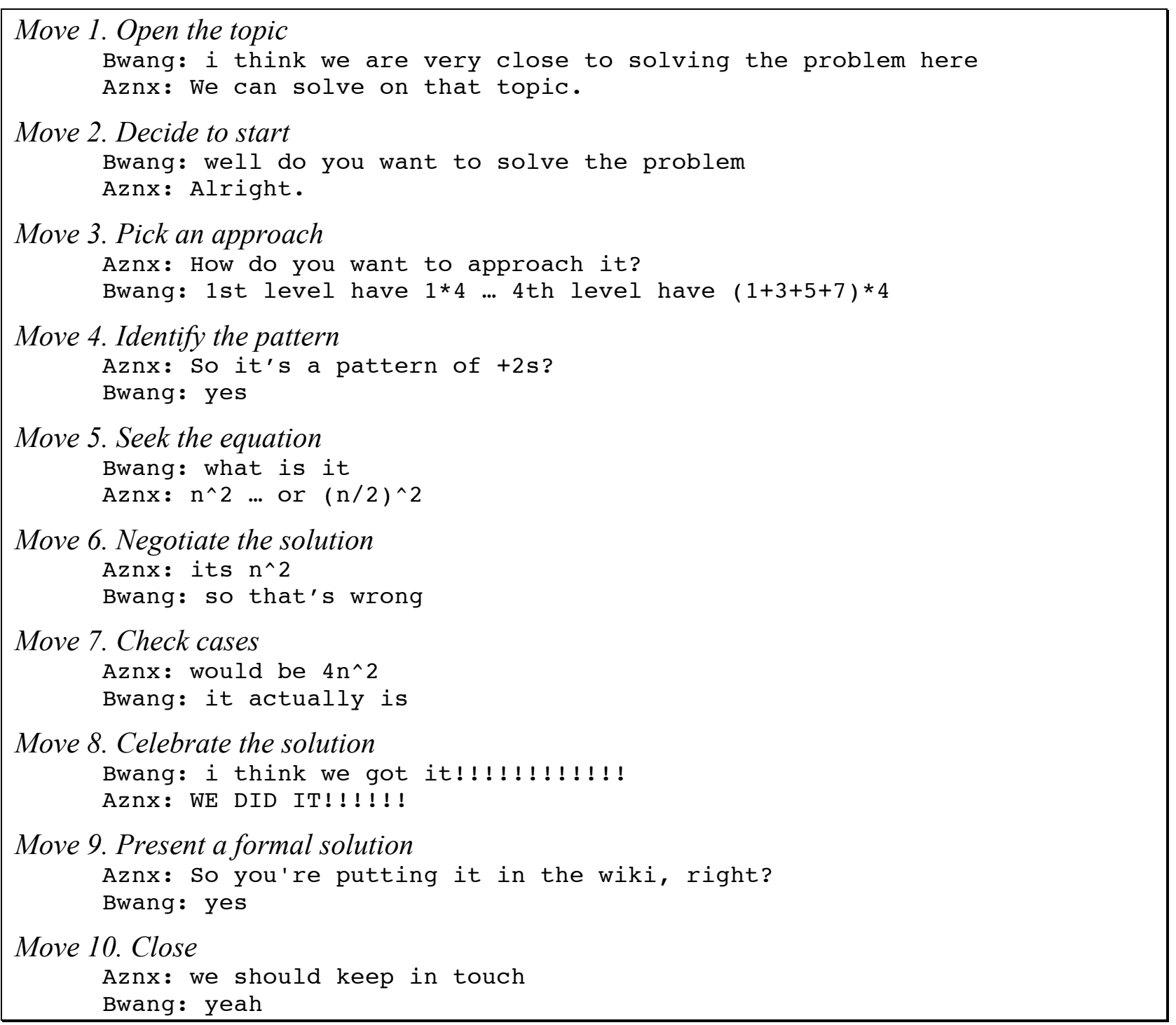

Log 1. Ten moves of the problem-solving topic.

There are several things to note here:

- Most importantly, the sequence of moves is strikingly similar to how an experienced math problem solver might approach the topic individually, as described at a particular granularity.

- The two students take turns contributing to the shared topic. The group direction is not set by either individual, but results from their interaction.

- Most opening utterances solicit a response, often in the explicit form of a question, and they always await a response.

- Each move is a situated response to the current state of the students' understanding of the topic as expressed in the discourse - rather than some kind of logical progression following a plan based on some kind of goal-subgoal hierarchy (Suchman, 2007). 
- The focus of the group discourse moves is on the sharing, negotiation and agreement about their progress, rather than on details of mathematical facts or computations.

- The math content is handled by the individuals and contributed by them into the collaborative setting, for instance in move \#3 or \#5.

- The temporal structure of topics, moves and adjacency pairs is not imposed by the analyst, but is projected in the remarks of the participants as integral to how they make meaning for themselves about what they are doing.

If one follows the development of the students' understanding in their postings across the four sessions, one is struck by changing roles and confidence levels, as well as by their mastery of practices that one or the other introduced into the group. It is quite plausible that over time the lessons acquired in their collaborative interactions become manifested in their individual cognitive skills. The longer sequences of argumentation or problem solving become "internalized" (as Vygotsky called it) or adopted as cognitive practices of individuals. The power of collaborative learning is partially to bring together multiple perspectives, which can be debated, negotiated, synthesized, contextualized, structured and refined. However, another advantage is to extend the cognitive effort into longer sequences of argumentation through the stimulation and enjoyment of productive social interaction, increasing the time-on-task as needed to solve challenging problems. Thus, groups can achieve cognitive accomplishments that their members cannot — and the members can learn from these achievements.

\section{VISUALIZING HEXAGONS}

Elsewhere, we have analyzed in some detail the intimate coordination of visual, narrative and symbolic activity involving the text-chat and shared whiteboard in VMT sessions (Çakir \& Stahl, 2013; Çakir, Zemel \& Stahl, 2009). Here, we want to bring out the importance of literally looking at some mathematical object together in order to share the visual experience and to relate to - to intend or to "be at"- the entity together. People often use the expression "I do not see what you mean" in the metaphorical sense of not understanding what someone else is saying. In our second case study, we often encounter the expression used literally for not being able to visually perceive a graphical object, at least not being able to see it in the way that the speaker apparently sees it.

While empiricist philosophy refers to people taking in uninterpreted sense data much like arrays of computer pixels, post-cognitive philosophy emphasizes the phenomenon of "seeing as." Wittgenstein noted that one immediately sees a wire-frame drawing of a cube not as a set of lines, but as a cube oriented either one way or another (1953: §177). For Heidegger, seeing things as already meaningful is not the result of cognitive interpretation, but the precondition of being able to explicate that meaning further in understanding (1927/1996, p. 139f.). For collaborative problem solving and mathematical deduction, it is clearly important that the participants see the visual mathematical objects as the same, in the same way. This seems to be an issue repeatedly in the online session excerpted in Log 2, involving three high-school students with login handles of Jason, Qwertyuiop and 137 (Stahl et al., 2011). 


\begin{tabular}{|l|l|l|l|}
\hline 705 & $19: 15: 08$ & 137 & $\begin{array}{l}\text { So do you want to first calculate the number of } \\
\text { triangles in a hexagonal array? }\end{array}$ \\
\hline 706 & $19: 15: 45$ & qwertyuiop & What's the shape of the array? a hexagon? \\
\hline 707 & $19: 16: 02$ & 137 & Ya. \\
\hline 708 & $19: 16: 15$ & qwertyuiop & ok... \\
\hline 709 & $19: 16: 41$ & Jason & $\begin{array}{l}\text { wait-- can someone highlight the hexagonal array } \\
\text { on the diagram? i don't really see what you } \\
\text { mean... }\end{array}$ \\
\hline 710 & $19: 17: 30$ & Jason & hmm. okay \\
\hline 711 & $19: 17: 43$ & qwertyuiop & oops \\
\hline 712 & $19: 17: 44$ & Jason & so it has at least 6 triangles? \\
\hline 713 & $19: 17: 58$ & Jason & in this, for instance \\
\hline
\end{tabular}

$\log 2$. Seeing a hexagonal array collaboratively.

Student 137 proposes a mathematical task for the group in line 705 of Log 2. This is the first time that the term, "hexagonal array," has been used. Coined in this posting, the term will become sedimented (Husserl, 1936/1989: 164) as a mathematical object for the group as the discourse continues. However, at this point it is problematic for both Qwertyuiop and Jason. In line 706, Qwertyuiop poses a question for clarification and receives an affirmative, but minimal, response. Jason, unsatisfied with the response, escalates the clarification request by asking for help in seeing the diagram in the whiteboard as a "hexagonal array," so he can see it as 137 sees it. Between Jason's request in line 709 and acceptance in line 710, Qwertyuiop and 137 work together to add lines outlining a large hexagon in the triangular array. Demonstrating his ability to now see the hexagons, Jason thereupon proceeds with the mathematical work, which he had halted in the beginning of line 709 in order to keep the group aligned. Jason tentatively proposes that every hexagon "has at least 6 triangles" and he makes this visible to everyone by pointing to an illustrative small hexagon from the chat posting, using the VMT graphical pointing tool. Later, the students take turns using these group-defined methods of supporting shared vision and attention: using colored lines and the pointing tool, as seen in Figure 2.

Jason dramatically halted group work with his "wait." For him, it was impossible to continue until everyone could see the same thing in the way that 137 saw it. During this session, the students taught each other how to change the color and thickness of lines they constructed in the shared whiteboard. These were affordances of the VMT software, but the students had to learn how to use the features and they developed certain shared group practices of using colored lines to outline, highlight and draw attention to specific elements of the hexagonal grid. For instance, in Figure 2, blue lines outline a hexagon of side length 3; red lines divide that hexagon into six symmetric triangles; thick green lines pick out the three horizontal lines of length 1, 2 and 3 in one of the triangles; and the VMT pointing tool focuses attention on that triangle. There are many ways to count the number of unit sticks in the large hexagon. In order to count them as a group, everyone's attention must be focused on the same elements, such as the green horizontals. Then it is possible for each participant to count that subset visually: $1+2+3=6$. Through similar shared attention to structural elements of the hexagon, all the group members know that there are three such arrays of lines like the green ones at different orientations in each of the six triangles. They can also see how this array of lines will increase as the hexagon itself progresses to successively longer side lengths. The achievement of the necessary persistent co-attention to construct and to follow this complicated analysis was the result of subtle interactions and the development of shared practices within the group. 


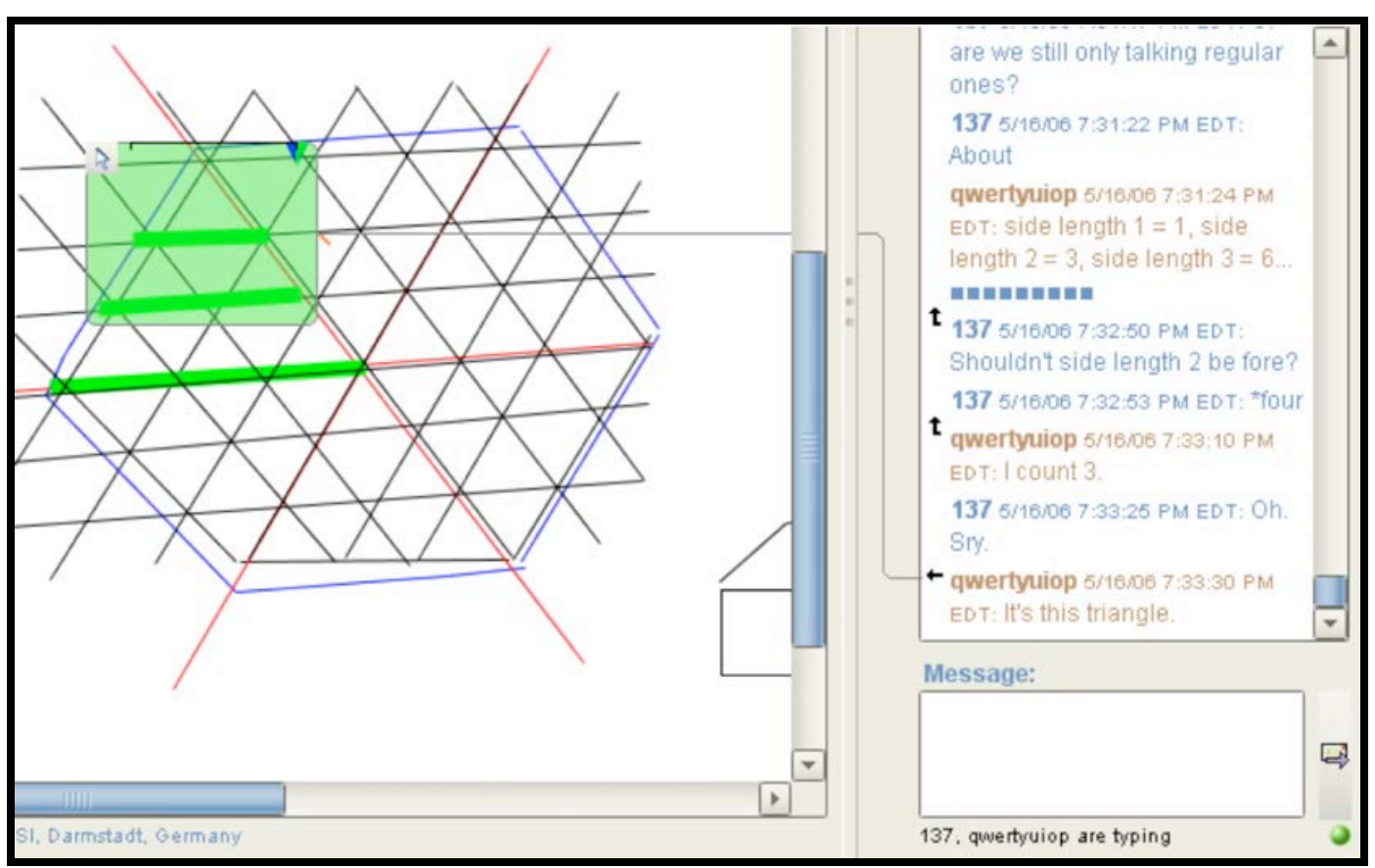

Figure 2. Discussion and drawing of hexagon grid.

\section{INSCRIBING TRIANGLES}

Our final case involves a group of three middle-school students given a topic in dynamic geometry (Stahl, 2013, §7.3). The students have not yet had a course in geometry, but have already spent four hours together in a version of VMT that incorporates interactive, multi-user support for dynamic geometry. In this topic, the students are given constructions of an equilateral triangle inscribed inside another equilateral triangle and a square inscribed inside another square (see Figure 3). In dynamic geometry, a student can drag one point of a figure like the inscribed squares and all the other points and lines will move accordingly, maintaining the geometric relationships or dependencies that have been built into the construction of the figure. In previous sessions, the students had learned the dynamic-geometry equivalent of Euclid's first two propositions: the construction of an equilateral triangle (using software tools equivalent to a straight edge and compass) and the copying of a line-segment length.

In their fifth session, the three students took turns dragging points of the equilateral triangles and discussing the dependencies that were maintained. Then they tried to duplicate the given figure and to build in the relevant dependencies. For instance, the dependency defining the equilateral character of the outer triangle is that the lengths of the second and third sides must always be the same as the length of the base, even when the end points of the base segment are dragged, changing its length. Euclid's construction maintains this dependency because the lengths of all three sides are radii of circles of equal radius. Read today, Euclid's Elements (300 BCE/2002) in effect provides instructions for dynamic-geometry constructions. The "elements" of geometry are not so much the points, lines, circles, triangles and quadrilaterals, but the basic operations of constructing figures with important relationships, such as congruence or symmetry. 
Just as Euclidean geometry contributed significantly to the development of logical, deductive, apodictic cognition in Western thought and in the formative minds of many prospective mathematicians, so collaborative experiences with dynamic geometry may foster in students ways of thinking about dependencies in the world.

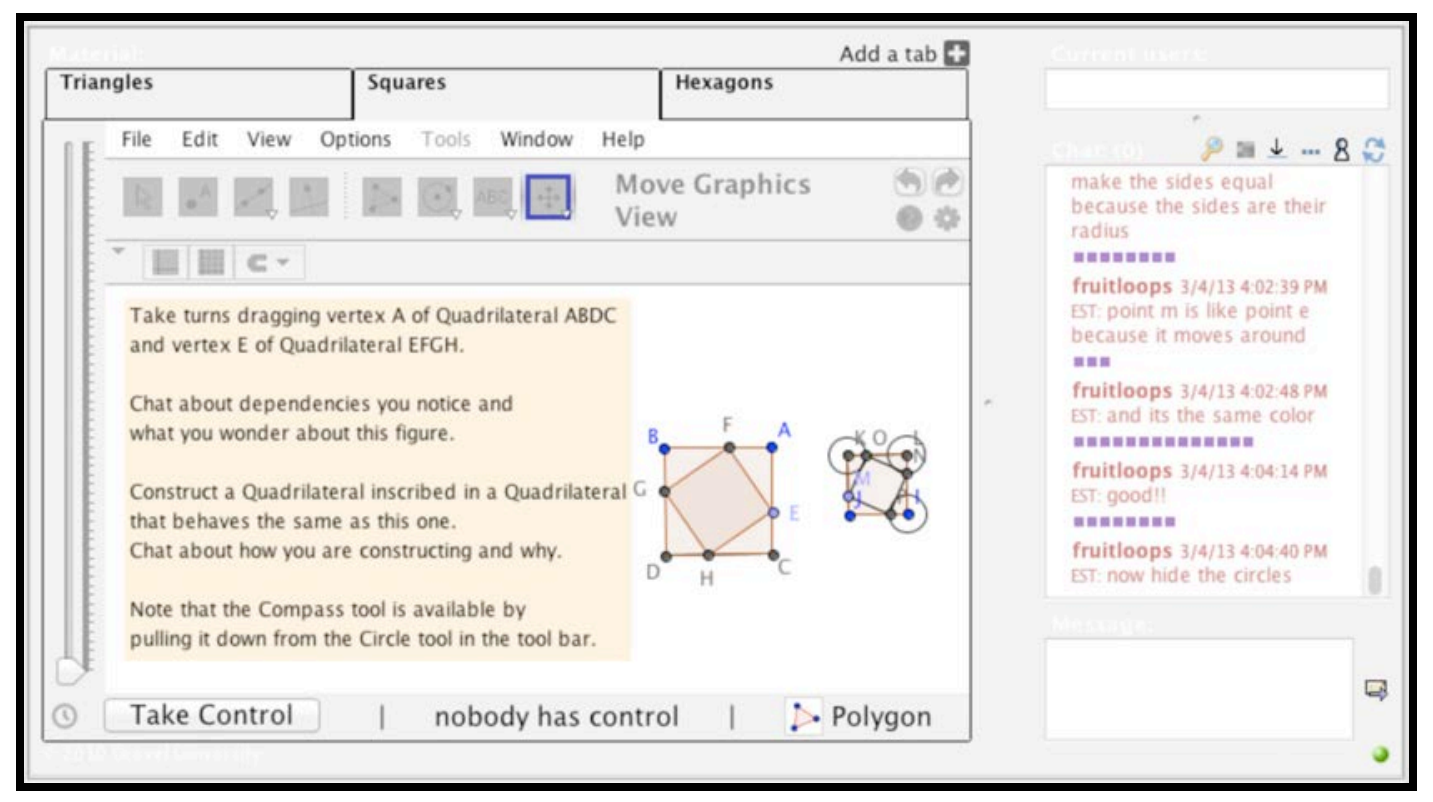

Figure 3. Discussion and constructions of inscribed squares.

The students in the case study used Euclid's method to construct the outside triangle, but soon realized that the same procedure could not be used to construct the inscribed triangle, because of the additional constraint that its vertices all had to be on the sides of the inscribing triangle, which they had constructed. Considerable further dragging of points in the given figure and experimentation with various construction approaches were tried. Finally, the students noticed that when one point of the inner triangle was dragged along a side of the outer triangle, the other vertices of the inner triangle moved in a corresponding way, such that their positions along their sides of the outer triangle were the same as that of the dragged vertex on its side. Then they quickly decided to use the method they had learned for copying a line-segment length. They copied the length from one outer vertex of their new equilateral triangle to a point for an inner vertex. Then they placed this length along the other sides, starting at both of the other vertices. This determined the locations of the other inner vertices. When they connected the three points, they formed an inscribed triangle. When any point or line was dragged, both the inner and outer triangles remained equilateral and inscribed.

In their sixth session, the students tackled the topic of inscribed squares. All their previous work in dynamic geometry had involved triangles and they had not been exposed to a method of constructing a dynamic square. They spent most of the hour exploring possible construction methods, eventually inventing a method that was elegantly similar to that of the triangle construction. All three students then immediately saw how to construct the interior square by copying the length from a corner of the exterior square to a corner of the interior one along a side. In Figure 3, the circles used for copying the length are still visible. The clarity with which 
each of the students understood how to inscribe a square-once they were able to construct the exterior dynamic square - shows how well they had each individually mastered the technique from their prior collaborative experience involving the dynamic triangles.

Their collaborative solution of the inscribed-triangles topic is quite typical. We have observed a number of small groups working on this topic, including math teachers, researchers, graduate students and middle-school students. They all go through a similar process of dragging the original figure, experimenting with construction attempts, discovering the dependency of the distances between the interior and exterior vertices, then realizing how to copy that distance and finally checking that their construction has the same behavior as the given figure. While this topic poses a problem that is difficult for individuals, small groups tend to stick with it and solve it through collaborative effort within an hour or less. It takes a combination of many trials, observations and connections to accomplish the task. The collaborative approach allows individuals to contribute specific pieces of the puzzle, to build on each other's proposals and to discuss the implications.

The chat discourse is striking in how much the students make sure that everyone agrees with and understands each step that the group as a whole takes in constructing their figures. In addition to expressing agreement and affirming understanding, the students also demonstrate their shared understanding by fluidly building on each other's contributions. Successive steps

are generally taken by different students, indicating that they are all following the logic of the collaborative effort.

\section{CONTRIBUTING TO GROUP COGNITION}

The cognition in group cognition is not the same as individual cognition; it relies upon individual cognition to make essential contributions. However, one cannot say that all of the cognition should be analyzed at the individual unit, because the work of assembling the high-level argumentative structure occurs at the group unit of analysis. Surely, putting together problemsolving arguments must be considered a cognitive activity as much as the work that goes into making the detailed contributions to individual steps. In addition, the personal contributions are largely responses to what has gone before in the group interaction. Not only are these contributions expressions that would not have occurred without the preceding opening up for them and elicitation of them by the group process, but many of the contributions are largely reactions at the group level, which reference and interrelate resources available in the discourse context more than they introduce new elements from the personal perspective and individual background of the actor. The important cognitive achievement is emergent at the group level, rather than a simple collection of expressions of individual cognitive accomplishments.

Coherent and impressive examples of group cognition-such as solving a math problem that the group members would not have been able to solve on their own - do not occur whenever a number of people come together in conversation. In fact, the research field of computersupported collaborative learning has documented that desirable forms of collaborative knowledge building are hard to find. The three studies summarized above indicate some reasons for this. First, it is difficult to set up a group interaction where everything relevant to the cognition at the group level of analysis is captured in a form adequate for detailed analysis. It took years of research to develop and deploy the VMT environment to successfully generate 
adequate data for the analysis of group cognition. Secondly, the group interaction must be directed and guided to focus on an appropriate cognitive task. Certain challenging math problems, carefully presented, seem to provide effective resources for stimulating interesting episodes of group cognition. Additionally - as the three studies summarized here have documented - the groups must work consistently to ensure the presence of certain preconditions of effective group cognition. They must persist in building longer sequences of responses to each other, they must maintain continuous co-attention to a shared focus of discussion and they must build and sustain a shared understanding of the topic of conversation.

\section{THE CONSTITUTION OF GROUP COGNITION}

The phenomenological tradition has always conceived of cognition as embodied in the world, rather than as a Cartesian mental process. Husserl (1929/1960: §14) emphasized that cognition is cognition of something; it is located at its object, not at some internal representation of that external object. Heidegger (1927/1996) therefore started from the experience of being-in-theworld instead of thinking-in-the-head. For him, cognition is a matter of being-with and caring-for things and people. The world is a shared world and the things we are there with are always already understood as meaningful. In Merleau-Ponty's (1945/2002) famous example of the blind man with the cane, the cane does not so much augment or extend the man's senses and awareness of external reality as it locates his cognition in the world at the tip of the cane.

If we look at the presented examples of group cognition, we see that the students are "there" in their group interaction with mathematical objects, seen in specific ways. Aznx and Bwang have drawn the horizontal sticks and the vertical sticks separately (not shown in the summary above). They have noticed a four-way symmetry, which allows them to reduce the problem of counting the sticks to a tractable pattern. They are focused together on the diamond as that symmetric pattern of sticks. Similarly, Jason, Qwertyuiop and 137 have worked hard to view their hexagonal array as a symmetrical pattern of sticks forming lines within triangles that make up a hexagon. As these groups work out their algebraic solutions to the topic, they are present together in a shared world at an object of interest, which they all see as structured in the same way. In the third case, after much work individually and collaboratively, and incorporating ideas from the ancient tradition of Euclidean geometry, the three students working on the inscribed squares all observe that when square $\mathrm{EFGH}$ is dragged within square $A B C D$ the following segments along the outer square change but stay equal in length to each other: $\mathrm{AE}, \mathrm{CH}, \mathrm{DG}, \mathrm{BF}$. They then can all see that they have to construct square MONP within square IJKL so that segments IP, JM, Ko, LN stay the same (see Figure 3). They collaborate in a shared world, manipulating a shared object physically, visually and imaginatively within a shared understanding of their problem, the geometric objects, the dynamic dependencies, the representational figure and the software affordances.

Following the phenomenologists, the ethnomethodologists showed that the shared social world is constituted continuously through group interaction (Garfinkel, 1967). In our VMT data, we can study precisely how that is accomplished. We see that it takes place over longer sequences of discourse moves, each centered on elicitation/response adjacency pairs. Carrying out these longer sequences requires maintaining persistent co-attention to a shared object; the being-there-together at the object provides a shared focus for the discourse. Accompanying this, there must be a shared understanding of the object and of the discourse context so that group 
members understand each other. If someone does not know what someone else means by a "hexagonal array" or by its "side-length," does not see the same elements of a symmetrical pattern or the same set of line segments moving together, then the collaborative problem solving cannot continue productively.

Kant (1787/1999) argued that the human mind constitutes meaningful reality through a process of creative discovery, in which structure is imposed to create and discover objects in the world. In the preceding examples, we see how group interaction can constitute the character of objects in the shared world and we have suggested that the shared meaningful world is itself constituted through such interaction. The nature of reality-such as the symmetries of diamond patterns, hexagonal arrays and inscribed squares - is discovered through the creation of interpretive views of objects. Effective perspectives are constrained by reality, which is not knowable except through these views. The creation of perspectives at the level of group cognition shifts the constitutive role from Kant's individual cognition to group and social cognition. Like the students in the virtual math teams, we first learn to see things as others see them in group-cognitive processes (which generally incorporate culturally sanctioned approaches). Subsequently - due to the power of language (e.g. naming, verbal description) —we can be there with those objects (diamonds, hexagons, squares) when we are not physically (or virtually) present with them in a shared group setting. We can even "internalize" (to use Vygotsky's metaphor) our ability to be-there-with these meaningful objects in the internal speech of individual thought. However, the fact that introspection of adults discovers (and assumes) the existence of many individual mental objects does not mean that those objects were not at some point in our development internalized from group-cognitive experiences in community contexts. An adequate analysis of cognition should recognize the constitutive roles of group cognition and their integration with phenomena of individual and social cognition.

\section{REFERENCES}

Çakir, M. P., \& Stahl, G. (2013). The integration of mathematics discourse, graphical reasoning and symbolic expression by a virtual math team. In D. Martinovic, V. Freiman \& Z. Karadag (Eds.), Visual mathematics and cyberlearning. New York, NY: Springer. Web: http://GerryStahl.net/pub/visualmath.pdf.

Çakir, M. P., Zemel, A., \& Stahl, G. (2009). The joint organization of interaction within a multimodal CSCL medium. International Journal of Computer-Supported Collaborative Learning. 4(2), 115-149. Web: http://GerryStahl.net/pub/ijCSCL_4_2_1.pdf.

Euclid. (300 BCE/2002). Euclid's elements (T. L. Heath, Trans.). Santa Fe, NM: Green Lion Press.

Garfinkel, H. (1967). Studies in ethnomethodology. Englewood Cliffs, NJ: Prentice-Hall.

Hegel, G. W. F. (1807/1967). Phenomenology of spirit (J. B. Baillie, Trans.). New York, NY: Harper \& Row.

Heidegger, M. (1927/1996). Being and time: A translation of Sein und Zeit (J. Stambaugh, Trans.). Albany, NY: SUNY Press.

Husserl, E. (1929/1960). Cartesian meditations: An introduction to phenomenology (D. Cairns, Trans.). The Hague, Netherlands: Martinus Nijhoff. 
Husserl, E. (1936/1989). The origin of geometry (D. Carr, Trans.). In J. Derrida (Ed.), Edmund Husserl's origin of geometry: An introduction. (pp. 157-180). Lincoln, NE: University of Nebraska Press.

Kant, I. (1787/1999). Critique of pure reason. Cambridge, UK: Cambridge University Press.

Merleau-Ponty, M. (1945/2002). The phenomenology of perception (C. Smith, Trans. 2 ed.). New York, NY: Routledge.

Sacks, H. (1962/1995). Lectures on conversation. Oxford, UK: Blackwell.

Schegloff, E. A. (2007). Sequence organization in interaction: A primer in conversation analysis. Cambridge, UK: Cambridge University Press.

Stahl, G. (2006). Group cognition: Computer support for building collaborative knowledge. Cambridge, MA: MIT Press. Web: http://GerryStahl.net/elibrary/gc.

Stahl, G. (2009). Studying virtual math teams. New York, NY: Springer. Web: http://GerryStahl.net/elibrary/svmt.

Stahl, G. (2011). How a virtual math team structured its problem solving. Paper presented at the Connecting computer-supported collaborative learning to policy and practice: CSCL 2011 conference proceedings. Lulu: ISLS. Proceedings pp. 256-263. Web: http://GerryStahl.net/pub/cscl2011stahl.pdf.

Stahl, G. (2013). Translating Euclid: Creating a human-centered mathematics: Morgan \& Claypool Publishers. Web: http://gerrystahl.net/elibrary/euclid.

Stahl, G., Koschmann, T., \& Suthers, D. (2013). Computer-supported collaborative learning: An historical perspective. In R. K. Sawyer (Ed.), Cambridge handbook of the learning sciences, revised version. Cambridge, UK: Cambridge University Press. Web: http://GerryStahl.net/pub/chls2.pdf.

Stahl, G., Zhou, N., Cakir, M. P., \& Sarmiento-Klapper, J. W. (2011). Seeing what we mean: Coexperiencing a shared virtual world. In Connecting computer-supported collaborative learning to policy and practice: CSCL 2011 conference proceedings. (Vol. I, pp. 534541). Lulu: ISLS. Web: http://GerryStahl.net/pub/cscl2011.pdf.

Suchman, L. A. (2007). Human-machine reconfigurations: Plans and situated actions (2nd ed.). Cambridge, UK: Cambridge University Press.

Vygotsky, L. (1930/1978). Mind in society. Cambridge, MA: Harvard University Press.

Wittgenstein, L. (1953). Philosophical investigations. New York, NY: Macmillan.

\section{Note on Contributor}

Gerry Stahl is Research Full Professor at the School of Information Science and Technology, Drexel University, Philadelphia, USA. He directs the Virtual Math Teams Project at the Math Forum and is founding editor of the International Journal of Computer-Supported Collaborative Learning. 